\title{
Deterministic dense coding with partially entangled states
}

\author{
Shay Mozes, ${ }^{1}$ Jonathan Oppenheim, ${ }^{2,3}$ and Benni Reznik ${ }^{1}$ \\ ${ }^{1}$ Department of Physics and Astronomy, Tel-Aviv University, Tel Aviv 69978, Israel \\ ${ }^{2}$ Department of Applied Mathematics and Theoretical Physics, University of Cambridge, Cambridge, United Kingdom \\ ${ }^{3}$ Racah Institute of Theoretical Physics, Hebrew University of Jerusalem, Givat Ram, Jerusalem 91904, Israel
}

(Received 26 March 2004; published 7 January 2005)

\begin{abstract}
The utilization of a $d$-level partially entangled state, shared by two parties wishing to communicate classical information without errors over a noiseless quantum channel, is discussed. We analytically construct deterministic dense coding schemes for certain classes of nonmaximally entangled states, and numerically obtain schemes in the general case. We study the dependency of the maximal alphabet size of such schemes on the partially entangled state shared by the two parties. Surprisingly, for $d>2$ it is possible to have deterministic dense coding with less than one ebit. In this case the number of alphabet letters that can be communicated by a single particle is between $d$ and $2 d$. In general, we numerically find that the maximal alphabet size is any integer in the range $\left[d, d^{2}\right]$ with the possible exception of $d^{2}-1$. We also find that states with less entanglement can have a greater deterministic communication capacity than other more entangled states.
\end{abstract}

DOI: 10.1103/PhysRevA.71.012311

PACS number(s): 03.67.Hk, 03.65.Ud, 03.67.Mn

\section{INTRODUCTION}

Dense coding, originally introduced by Bennett and Wiesner [1], is the surprising utilization of entanglement to enhance the capacity of a quantum communication channel. Two parties, Alice and Bob, communicate by sending a spin$\frac{1}{2}$ particle (a qubit) over a noiseless quantum channel. As no more than two spin states can be perfectly distinguished, Alice can encode only one of two different letters, say 0 or 1 , within each particle she sends. This is no better than using a classical communication channel. However, Bennett and Wiesner have shown that if Alice and Bob each have one particle of a maximally entangled pair, it is possible for the sender Alice to transform the two-particle state into four orthogonal states by acting locally on her particle. After sending Bob her half of the pair, he will be able to distinguish the four different states perfectly by measuring the pair of particles collectively. Surprisingly, this enables the transmission of one of four letters by sending a single qubit, provided that the two parties share initial entanglement.

Numerous aspects of dense coding have been studied. Among these are generalizations to pairs of entangled $d$-level systems [1], to continuous variables [2], and to settings involving more than two parties [3]. Other works [4-6] studied dense coding in the asymptotic limit, where many copies of a partially entangled state are used.

In this paper we consider the case of pure nonmaximal entanglement between two separated $d$-level systems. We are not interested in the asymptotic channel capacity, but rather in the deterministic procedure, where the parties wish to distinguish without errors messages encoded by acting only on a single $d$-level particle. We use exact and numerical methods to study the relation between a state $|\psi\rangle$, whose entanglement is given by its entropy $S(\psi)$, and $N_{\max }(\psi)$, the maximal size of the alphabet which can be perfectly communicated. In other words, $N_{\max }(\psi)$ denotes the maximal number of orthogonal states that can be generated by means of a unitary transformation acting locally on Alice's part of the given entangled state.
Our results suggest that for a dimension $d>2$, deterministic dense coding processes which utilize partially entangled states are possible for any maximal alphabet size $N_{\max }(\psi)$ in the range $\left[d, d^{2}\right]$ with the possible exception of $d^{2}-1$. Since the total dimension of Alice and Bob's state is $d \times d$, this appears to be an interesting boundary effect. The maximal alphabet size $N_{\max }=d^{2}$ is only possible with maximally entangled states. Using numerical methods, the existence of all values except $d^{2}-1$ has been fully verified for $d=3$ (see Fig. 1) and $d=4$, and partially for $d=5, \ldots, 7$. Analytically, we have been able to construct dense coding schemes for an alphabet size that is a multiple of $d$ (i.e., $k d, k=2, \ldots, d-1$ ), and for an alphabet size of $d+1$.

We have found (both analytically and numerically) that there are states with less than one ebit of entanglement that can be used for deterministic dense coding, although in this case the maximal alphabet size is less than $2 d$, where $d$ is the dimension. Therefore, our method is not equivalent to the trivial approach wherein deterministic concentration transforms a nonmaximal state, which must have more than one ebit of entanglement, into a single ebit [11], to be used in the standard dense coding scheme.

In addition, we find that entanglement, while playing an important role in the communication capacity, does not completely determine $N_{\max }(\psi)$. We numerically find that one can have two states with the property that the less entangled one is in fact better for deterministic communication. That is, we can have $N_{\max }\left(\psi_{1}\right)>N_{\max }\left(\psi_{2}\right)$ while $S\left(\psi_{1}\right)<S\left(\psi_{2}\right)$. This is perhaps also interesting in light of [7] where it was shown that states with less entanglement sometimes have a greater probability of being distinguished by separated parties who can only communicate classically. A related situation has been reported in [8] wherein nonmaximal states, rather than maximal, were needed to perform certain remote operations.

This paper is organized as follows. We first review deterministic dense coding with maximally entangled $d$-level systems. Then we proceed to formulate the problem considered in this paper. Section IV treats the two-dimensional case ana- 


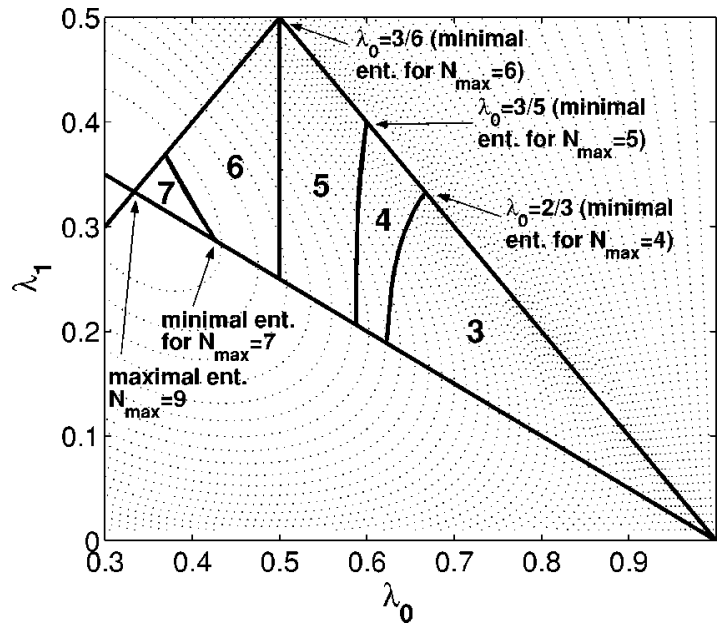

FIG. 1. Numerical mapping of $N_{\max }(\psi)$, the maximal number of orthogonal unitaries with respect to $|\psi\rangle$ over the domain of pure states of two qutrits $|\psi\rangle=\sqrt{\lambda_{0}}|00\rangle+\sqrt{\lambda_{1}}|11\rangle+\sqrt{1-\lambda_{0}-\lambda_{1}}|22\rangle$. The horizontal axis is $\lambda_{0}$ and the vertical axis is $\lambda_{1}$. The region of interest is defined by $\lambda_{1} \leqslant \lambda_{0}, \lambda_{0}+\lambda_{1} \leqslant 1$, and $\lambda_{1} \geqslant\left(1-\lambda_{0}\right) / 2$. This region was found to be divided into five subregions characterized by different values of $N_{\max }(\psi)$. Contour lines of the entanglement, $S(\psi)$, are plotted in the background. It is evident that $S(\psi)$ does not determine $N_{\max }(\psi)$. There are many states $|\psi\rangle$ and $|\phi\rangle$ having $S(\psi)>S(\phi)$, but $N_{\max }(\psi)<N_{\max }(\phi)$. States with minimal entanglement, admitting at least $N$ orthogonal unitaries $(N=4,5,6,7)$, are indicated in the figure. Note that no region with eight unitaries was found. The only case where nine unitaries exist is the maximally entangled state $\left(\lambda_{0}=\lambda_{1}=1 / 3\right)$.

lytically and shows that nonmaximal states cannot be used to distinguish perfectly more than two letters. In Sec. V we present exact forms for two different kinds of deterministic dense coding schemes in dimensions $d>2$. In Sec. VI our numerical results are presented and discussed. Finally, we summarize the results in Sec. VII.

\section{DENSE CODING WITH MAXIMAL ENTANGLEMENT}

We consider a bipartite qudit pure state. That is, a system composed of two $d$-level separated subsystems. This system is initially prepared in a maximally entangled state

$$
\left|\psi_{00}\right\rangle=\frac{1}{\sqrt{d}} \sum_{i=0}^{d-1}|i\rangle_{A} \otimes|i\rangle_{B},
$$

where $A(B)$ denotes Alice's (Bob's) subsystem. Alice encodes an alphabet of size $d^{2}$, which we denote as $\{(m, n)\}_{m, n=0}^{d-1}$, using a set $\left\{U_{m n}^{A}\right\}$ of local unitary operations on particle $A$. There are many possible [10] realizations of this set of operators. An elegant, and undoubtedly the most common, construction is

$$
U_{m n}=(X)^{m}(Z)^{n},
$$

where $X$, the shift operator, and $Z$, the rotate operator, are defined by

$$
X|k\rangle=\left|(k+1)_{\bmod (d)}\right\rangle,
$$

$$
Z|k\rangle=e^{(2 \pi i k / d)}|k\rangle .
$$

It can easily be verified that $\left|\psi_{m n}\right\rangle=\left(U_{m n}^{A} \otimes \mathbb{1}_{B}\right)\left|\psi_{00}\right\rangle$ form an orthogonal basis of the two-qudit Hilbert space. After encoding the letter $(m, n)$, Alice sends her particle to Bob through the quantum channel. Bob performs a projective measurement of the two-particle state on $\left\{\left|\psi_{m n}\right\rangle\right\}$ to decode the message.

A few remarks are in order here. First, we note that for qubits $(d=2)$, this basis is just the well known Bell basis:

$$
\begin{aligned}
& \left|\psi_{00}\right\rangle=\frac{1}{\sqrt{2}}(|00\rangle+|11\rangle), \quad\left|\psi_{01}\right\rangle=\frac{1}{\sqrt{2}}(|00\rangle-|11\rangle), \\
& \left|\psi_{10}\right\rangle=\frac{1}{\sqrt{2}}(|10\rangle+|01\rangle), \quad\left|\psi_{11}\right\rangle=\frac{1}{\sqrt{2}}(|10\rangle-|01\rangle) .
\end{aligned}
$$

Second, trying to understand intuitively the difference between the classical and quantum cases, we note that the shift operators may be regarded as "classical," in the sense that they correspond to the possibility of sending $d$ distinct values of a classical dit. The rotate operators may be regarded as the quantum enhancement, which enables the local realization of $d^{2}$ orthogonal two-qudit states.

\section{DETERMINISTIC DENSE CODING WITH NONMAXIMAL ENTANGLEMENT}

We now introduce the main problem this paper addresses. Instead of using a maximally entangled state, we consider an arbitrary bipartite pure state. This can be written in the Schmidt representation [9] as

$$
|\psi\rangle=\sum_{i=0}^{d-1} \sqrt{\lambda_{i}}|i\rangle_{A} \otimes|i\rangle_{B}, \quad \sum_{i=0}^{d-1} \lambda_{i}=1,
$$

where $|i\rangle_{A}\left(|i\rangle_{B}\right)$ are the Schmidt basis for system $A(B)$.

We are interested in a maximally sized set of local unitary operators $\left\{U_{i}^{A}\right\}_{i=0}^{N_{\max }(\psi)-1}$ that transform $|\psi\rangle$ into orthogonal states. That is, for all $0 \leqslant i, j<N_{\max }(\psi)$ we have

$$
\left\langle\psi\left|\left(U_{i}^{A^{\dagger}} \otimes \mathbb{1}_{B}\right)\left(U_{j}^{A} \otimes \mathbb{1}_{B}\right)\right| \psi\right\rangle=\delta_{i, j} .
$$

Substituting the state (5) into (6) yields

$$
\begin{aligned}
\delta_{i, j} & =\sum_{k, l=0}^{d-1} \sqrt{\lambda_{k} \lambda_{l}}\left\langle k\left|U_{i}^{\dagger} U_{j}\right| l\right\rangle\langle k \mid l\rangle=\sum_{k=0}^{d-1} \lambda_{k}\left\langle k\left|U_{i}^{\dagger} U_{j}\right| k\right\rangle \\
& =\operatorname{Tr}\left(\Lambda U_{i}^{\dagger} U_{j}\right)
\end{aligned}
$$

where $\Lambda$ is a $d \times d$ diagonal matrix of the Schmidt coefficients of $|\psi\rangle\left(\Lambda_{k k}=\lambda_{k}\right)$. Note that the matrices $U_{i}$ are unitary in the usual sense $\left(U_{i}^{\dagger} U_{i}=1\right)$, but the orthogonality of operators is now defined with respect to a nontrivial weight vector (the Schmidt coefficients), rather than the usual trace. For the rest of this paper orthogonality of operators should be understood in this sense.

In this paper our goal is to study the effect of the partially entangled state $|\psi\rangle$ on $N_{\max }(\psi)$, the maximal size of the set of unitaries satisfying (7). In other words, we would like to 
understand and characterize the relation $N_{\max }(\psi)$.

We first note that for any choice of $|\psi\rangle$, there always exists a set of at least $d$ such unitaries. This is the set of shift operators introduced in the previous section. Let us explicitly verify that orthogonality is indeed maintained:

$$
\begin{aligned}
& \left\langle\psi\left|\left(X_{A}^{n \dagger} \otimes \mathbb{1}_{B}\right)\left(X_{A}^{m} \otimes 1_{B}\right)\right| \psi\right\rangle \\
& \quad=\sum_{i, j} \sqrt{\lambda_{i} \lambda_{j}}\left\langle(i+n)_{\bmod (d)} \mid(j+m)_{\bmod (d)}\right\rangle\langle i \mid j\rangle=\delta_{n, m} .
\end{aligned}
$$

That this set is always orthogonal should not surprise us as it corresponds to the possibility of encoding $d$ distinct values in a single dit in the classical case.

\section{THE TWO-DIMENSIONAL CASE}

We begin by considering the case of partially entangled qubits $(d=2)$. We shall show that for all nonmaximally entangled states, only $N_{\max }(\psi)=2$ unitaries can be constructed. This means that deterministic dense coding with partial entanglement is not possible in $d<3$ dimensions; partially entangled qubits have no advantage over pure product states or classical bits.

For convenience, and without loss of generality, we assume that $l \in\left\{U_{i}\right\}$. We parametrize $U=e^{i \vec{\sigma} \cdot \hat{n} \theta}=\cos \theta 1$ $+i \sin \theta(\vec{\sigma} \cdot \hat{n})$, where $\vec{\sigma}$ are the Pauli matrices, and $\hat{n}$ is a unit vector. Since $1 \in\left\{U_{i}\right\}$, it follows from Eq. (7) that for all 1 $\neq U \in\left\{U_{i}\right\}, \operatorname{Tr}(\Lambda \rrbracket U)=0$. That is,

$$
0=\left(\lambda_{0}+\lambda_{1}\right) \cos \theta+i\left(\lambda_{0}-\lambda_{1}\right) \hat{n}_{z} \sin \theta
$$

which determines $\theta=\pi / 2$ and $\hat{n}_{z}=0$. Suppose we want to have a set of three unitaries $\left\{1, U_{1}, U_{2}\right\}$. $U_{1(2)}$ must therefore be of the form $U_{1(2)}=i\left(\sigma_{x} x_{1(2)}+\sigma_{y} y_{1(2)}\right)$. Again, applying Eq. (7) with $i=1$ and $j=2, U_{1}$ and $U_{2}$ must satisfy

$$
\begin{aligned}
0= & \operatorname{Tr}\left(\Lambda U_{1}^{\dagger} U_{2}\right)=\left(\lambda_{0}+\lambda_{1}\right)\left(x_{1} x_{2}+y_{1} y_{2}\right) \\
& +i\left(\lambda_{0}-\lambda_{1}\right)\left(x_{1} y_{2}-y_{1} x_{2}\right) .
\end{aligned}
$$

For nonmaximal entanglement we have $\lambda_{0}-\lambda_{1} \neq 0$, and the normalization condition sets $\lambda_{0}+\lambda_{1}=1$. In addition we have $x_{1}^{2}+y_{1}^{2}=x_{2}^{2}+y_{2}^{2}=1$. Combining all these restrictions, Eq. (10) has no solutions. This proves that in the two-dimensional case, for a nonmaximally entangled state $|\psi\rangle, N_{\max }(\psi)=2$.

\section{HIGHER DIMENSIONS, EXACT SOLUTIONS}

In this section we show deterministic dense coding schemes for some classes of partially entangled states in $d$ $>2$ dimensions. First, we present a geometric approach for constructing dense coding schemes with an alphabet size of $N=k d$, where $k \leqslant d$ is an integer. The partially entangled states on which these schemes are based have all Schmidt coefficients not greater than $1 / k$. Next, we present a nongeometric approach for the explicit construction of deterministic dense coding schemes with an alphabet size of $d+1$. These schemes utilize the partially entangled state $\left|\psi_{d}\right\rangle$ $=\sqrt{(d-1) / d}|00\rangle+\sqrt{(1 / d)}|11\rangle+0 \sum_{i=2}^{d-1}|i i\rangle$. Note that these are states with less than one ebit of entanglement for $d>2$.

\section{A. The geometric approach}

Regarding the shift operators as "classical," and the rotate operators as the quantum enhancement, one may try to generalize the maximal dense coding scheme by constructing rotations, or phase operators, suitable for the given nonmaximal entanglement. (Recall that the shift operators need not be changed since they are orthogonal with respect to any state.) In analogy to (3), we are looking for a set $\left\{Z_{n}\right\}_{n=0}^{k-1}$ defined by

$$
Z_{n}|j\rangle=e^{i \theta_{j}^{n}}|j\rangle,
$$

where $0 \leqslant j<d, 0 \leqslant n<k \leqslant d$, and $\theta_{j}^{n}$ are real phases whose choice will be discussed shortly. The orthogonality requirement dictates that

$$
\delta_{m, n}=\left\langle\psi\left|Z_{n}^{\dagger} Z_{m}\right| \psi\right\rangle=\sum_{i, j} \sqrt{\lambda_{i} \lambda_{j}} e^{i\left(\theta_{j}^{n}-\theta_{i}^{n}\right)}\langle i i \mid j j\rangle=\sum_{j} \lambda_{j} e^{i\left(\theta_{j}^{n}-\theta_{j}^{n}\right)} .
$$

A set of $k$ such operators for a given state $|\psi\rangle$ can be used to construct $N=k d$ orthogonal operators [in the sense of (7)], namely, $U_{m n}=(X)^{m}\left(Z_{n}\right)$, where $0 \leqslant m<d$ and $0 \leqslant n<k \leqslant d$. In this construction the total number of operators is a multiple of $d$. In the classical or nonentangled case, it is $1 \times d$, and in the maximal case it is $d \times d$. As we will show in the following sections, this scheme is not an optimal one in the sense that there are other schemes using the same partially entangled state $|\psi\rangle$ with an alphabet size of $N_{\max }>N=k d$. This is why we denote the alphabet size $N(\psi)$ rather than $N_{\max }(\psi)$. However, $N(\psi)$ is a lower bound of $N_{\max }(\psi)$.

To examine the relation between the initial state $|\psi\rangle$ and $N(\psi)$, let us consider the simple case where we look for $k$ $=2$ phase operators. Again, we assume that $1 \in\left\{Z_{i}\right\}$, so that Eq. (12) reduces to $\sum_{i} \lambda_{i} e^{i \theta_{i}}=0$. In other words, we are faced with the geometric task of forming a polygon using $d$ vectors of lengths $\left\{\lambda_{0}, \lambda_{1}, \ldots, \lambda_{d-1}\right\}$. This can always be accomplished if the longest vector is shorter than the sum of the others. Assuming that the $\lambda_{i}$ 's are given in descending order, this condition is simply $\lambda_{0} \leqslant 1 / 2$. For the rest of the paper we will assume that the Schmidt coefficients are indeed given in descending order.

For the generalization to $k>2$ phase operators satisfying (12), we have no simple geometric interpretation. Similar phase factors were also used independently in the context of deterministic teleportation schemes [14]. It can be shown that such phases can only be found if $\lambda_{0} \leqslant 1 / k$. However, it is not known that this requirement is sufficient. As an example, for states in four dimensions whose Schmidt coefficients satisfy $1 / 3=\lambda_{0}=\lambda_{1}=\lambda_{2}+\lambda_{3} \quad(d=4)$, we can construct $N=3$ $\times 4=12$ operators by using products of the four shift operators, and the three phase operators defined by the phases $\theta_{0}^{m}=0, \theta_{1}^{m}=2 \pi m / 3, \theta_{2}^{m}=\theta_{3}^{m}=4 \pi m / 3$, where $0 \leqslant m<3$.

In order to prove [15] that $\lambda_{0} \leqslant 1 / k$ is a necessary condition for the existence of $k d$ phases satisfying Eq. (12), we define a $k \times d$ matrix $V$, whose elements are $V_{n j}=\sqrt{\lambda_{j}} e^{i \theta_{j}^{n}}$. From Eq. (12) it follows that $V V^{\dagger}$ is the $k$-dimensional identity matrix. Therefore, the $d$-dimensional Hermitian matrix $V^{\dagger} V$ has $k$ eigenvalues equal to 1 , and $d-k$ eigenvalues equal 
to zero. Note also that the diagonal elements of this matrix are given by $\left(V^{\dagger} V\right)_{j j}=k \lambda_{j}$. We now use a part of the SchurHorn theorem [16] which states that the vector of the diagonal elements of a Hermitian matrix is majorized by the vector of its eigenvalues. Therefore, in our case, the vector $\left(k \lambda_{0}, k \lambda_{1}, \ldots, k \lambda_{d-1}\right)$ is majorized by the vector of eigenvalues $(1,1, \ldots, 1,0, \ldots, 0)$. In particular, this proves that $\lambda_{0} \Leftarrow 1 / k$.

We note that states with $\lambda_{0} \leqslant 1 / k$ have entanglement $S$ $\geqslant \log _{2} k$ ebits. Furthermore, all such states are majorized by a maximally entangled state of a $k$-level system residing in a $d$-dimensional Hilbert space $\left[(1 / \sqrt{k}) \sum_{i=0}^{k-1}|i i\rangle+0 \sum_{i=k}^{d-1}|i i\rangle\right]$, and thus can be converted to it by local operations and classical communication $[11,12]$. For this "maximally" entangled state a construction similar to (3) trivially yields $k d$ orthogonal states. Note, however, that in order to concentrate $|\psi\rangle$ deterministically into a maximally entangled $k$-level state, one must use both local operations and classical communications [13], whereas in our construction only local operations are used. The additional communication required to convert nonmaximally entangled states into maximally entangled ones, would reduce the net gain in communication.

\section{B. A nongeometric approach}

As we have already mentioned, the geometric approach, although guided by the appealing separation into "classical" and quantum encoding operators, is not necessarily optimal. Consider, for example, the state

$$
\left|\psi_{3}\right\rangle=\sqrt{\frac{2}{3}}|00\rangle+\sqrt{\frac{1}{3}}|11\rangle+0|22\rangle
$$

in $d=3$ dimensions. Since $\lambda_{0}=2 / 3>1 / 2$, using the geometric approach we can only use the three operators $\left\{1, X, X^{2}\right\}$. This, however, does not mean that the maximal size of a set of orthogonal unitaries is just three. In fact, if we abandon the phase and shift operators, we find the larger set $\left\{1, X, U_{3}, U_{3}^{\dagger}\right\}$, where

$$
U_{3}=\left(\begin{array}{ccc}
-\frac{1}{2} & 0 & -\frac{\sqrt{3}}{2} \\
0 & 1 & 0 \\
\frac{\sqrt{3}}{2} & 0 & -\frac{1}{2}
\end{array}\right)
$$

is a rotation by $2 \pi / 3$ within the subspace spanned by $\{|0\rangle,|2\rangle\}$. This set consists of four orthogonal unitaries (with respect to $\left.\left|\psi_{3}\right\rangle\right)$. As will be discussed in the next section, our numerical results suggest that $\left|\psi_{3}\right\rangle$ is the state with minimal entanglement in $d=3$ dimensions admitting more than three orthogonal unitaries. Note that the above construction is by no means unique. It can be generalized to an arbitrary dimension $d$ as follows. The partially entangled state is

$$
\left|\psi_{d}\right\rangle=\sqrt{\frac{d-1}{d}}|00\rangle+\sqrt{\frac{1}{d}}|11\rangle+0 \sum_{i=2}^{d-1}|i i\rangle .
$$

The set of $d+1$ orthogonal unitaries is $\left\{\mathbb{1}_{d}, X\right\} \cup\left\{U_{d\}_{k=0}^{k}}^{d-2}\right.$, where

$$
\begin{gathered}
U_{d}^{k}|0\rangle=-\frac{1}{d-1}|0\rangle+\frac{\sqrt{d}}{d-1} \sum_{j=1}^{d-2} e^{2 \pi i k j /(d-1)}|j+1\rangle, \\
U_{d}^{k}|1\rangle=|1\rangle .
\end{gathered}
$$

The effect of $U_{d}^{k}$ on all other basis vectors is restricted only by the unitarity requirement $U_{d}^{k^{\dagger}} U_{d}^{k}=1$. Let us verify explicitly that $\left\{U_{d}^{k}\right\}$ is indeed an orthogonal set (we omit the subscript $d$ ):

$$
\begin{aligned}
\operatorname{Tr}\left(\Lambda U^{k^{\dagger}} U^{l}\right) & \left.\left.=\frac{d-1}{d}\langle 0| U^{k^{\dagger}} U^{l}\right)|0\rangle+\frac{1}{d}\langle 1| U^{k^{\dagger}} U^{l}\right)|1\rangle \\
& =\frac{1}{d(d-1)}\left(1+d \sum_{j=1}^{d-2} e^{2 \pi i(l-k) j((d-1)}\right)+\frac{1}{d} \\
& =\frac{1}{d-1}+\frac{1}{d-1} \sum_{j=1}^{d-2} e^{2 \pi i(l-k) j /(d-1)} \\
& =\frac{1}{d-1} \sum_{j=0}^{d-2} e^{2 \pi i(l-k) j /(d-1)}=\delta_{k, l}
\end{aligned}
$$

and also

$$
\begin{aligned}
\operatorname{Tr}\left(\Lambda \cdot 1 \cdot U^{k}\right) & =\frac{d-1}{d}\left\langle 0\left|U^{k}\right| 0\right\rangle+\frac{1}{d}\left\langle 1\left|U^{k}\right| 1\right\rangle \\
& =-\frac{d-1}{d} \frac{1}{d-1}+\frac{1}{d}=0, \\
\operatorname{Tr}\left(\Lambda X^{\dagger} U^{k}\right) & =\frac{d-1}{d}\left\langle 0\left|X^{\dagger} U^{k}\right| 0\right\rangle+\frac{1}{d}\left\langle 1\left|X^{\dagger} U^{k}\right| 1\right\rangle \\
& =\frac{d-1}{d}\left\langle 1\left|U^{k}\right| 0\right\rangle+\frac{1}{d}\left\langle 2\left|U^{k}\right| 1\right\rangle=0 .
\end{aligned}
$$

This construction can be further generalized to cases where $\lambda_{0}=d / N=(m-1) / m$ for some integer $m$. Note that all these states have less than one ebit of entanglement, and that for large $d, \lambda_{0}=(d-1) / d \simeq 1$, which means that the entanglement required for having more than the "classical" $d$ unitaries approaches zero.

\section{NUMERICAL ANALYSIS OF THE GENERAL CASE}

In the general case, we were unable to find a parametrization of Eq. (7) which leads to an exact solution. Numerical results are, however, obtainable. We first describe the numerical methods we have used, and some considerations regarding the reliability and accuracy of these results. Next, we present and discuss the numerical results we have obtained.

\section{A. Numerical methods and considerations}

To study this problem numerically, we have used standard numerical multivariate root-finding routines. These routines try to converge to a solution, starting from a given (usually random) point in the domain of parameters. The process either results in a set of values of the unknown parameters that 
is a root of the given multivariate function (with a certain controlled error), or fails to converge. Generally with such routines, a failure to converge means that the procedure got stuck in a local minimum, and that another trial with a different starting point may converge to a root. A consistent failure to converge over a sufficient number of trials suggests that the specified function has no roots. With our problem, for a given state $|\psi\rangle$ and a chosen alphabet size of $N$, it is possible to express the set of equations in (7) as a multivariate root-finding problem. The unknown parameters we wish to solve are those describing the unitaries $\left\{U_{i}\right\}_{i=0}^{N-1}$. Since the number of these parameters is quite large $\left(d^{2}-1\right.$ parameters per unitary), restarting the numerical procedure with random starting points a sufficient number of times in order to determine that there is no solution is not feasible. However, we noticed that for any choice of $|\psi\rangle$ and $N$ only one of two cases occurs. Either the procedure finds a solution for any given starting point (not necessarily the same solution for different starting points, but it always finds a solution), or no solution is found for all given starting points. This was checked for many choices of pure states and sizes of alphabets, using 100 random starting points for each choice. This fact indicates that the two cases differ substantially in the number and density of solutions. Therefore, when performing a systematic, high-resolution mapping of the domain of pure states and sizes of alphabet we used a single random starting point to determine whether dense coding is possible for a choice of $|\psi\rangle$ and $N$ or not. For a small set of values of $|\psi\rangle$ and $N$ which are of special interest, and will be described later on, we did use several starting points to enhance the reliability of our results. To confirm our results we have verified that in all cases where we know that deterministic dense coding is possible (i.e., maximal entanglement and the cases discussed in the previous section), the numerical procedure indeed found a solution. Furthermore, the fact that our results, which will be described in detail shortly, demonstrate the existence of well defined regions with smooth boundaries indicates that the our numerical analysis captured the true properties of this system, and not some random artifacts.

\section{B. Numerical results}

Using the method described above, we have mapped the entire domain of pure states in three dimensions and some regions of the domain of pure states up to dimension $d=7$. The increasing nature of both the number of parameters describing the unitaries, and the size of the domain of pure states, makes an exhaustive mapping in high dimensions extremely time consuming. Figure 1 graphically presents the results for the three-dimensional case. It is evident that one can have two states $|\psi\rangle$ and $|\phi\rangle$ having $S(\psi)>S(\phi)$ but $N_{\max }(\psi)<N_{\max }(\phi)$. Naively, one may expect more entanglement to mean greater deterministic communication capacity, yet this is not so. Such cases were also found in our partial mappings of higher dimensions and have led us to conclude that in finite dimensional systems, $N_{\max }(\psi)$, the maximal number of orthogonal unitaries, does not depend directly on the entanglement, but on some other function of the Schmidt coefficients $\lambda_{i}$. We attribute this to the fact that the entangle-

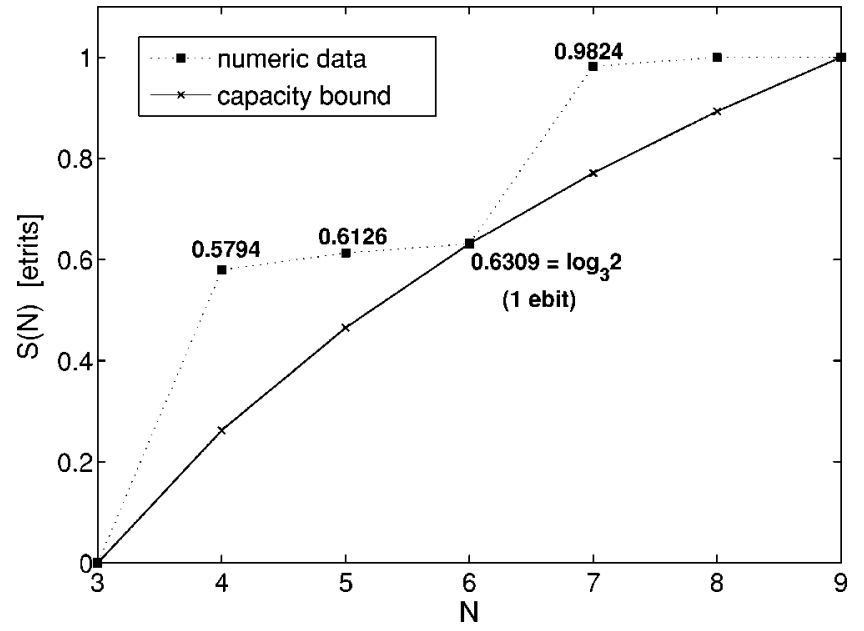

FIG. 2. Minimal entanglement (in etrits) required to construct $N$ orthogonal unitaries as a function of $N$ in the three-dimensional case. Numerical results are shown as black squares connected by dotted lines. These entanglement values were extracted from the minimally entangled states indicated in Fig. 1. $\times$ symbols connected by solid lines indicate the channel capacity bound.

ment measure (the von Neumann entropy) is an asymptotic quantity, while the process we consider here can be carried out deterministically with a single entangled pair.

An intriguing observation is that we did not find partially entangled pure states for which it is possible to construct a maximal set of eight orthogonal unitaries (but we did find all steps with $N_{\max } \leqslant 7$ ). Similarly, we have not found any pure state in $d=4$ dimensions, admitting the construction of a maximal set of 15 orthogonal unitaries (and again, we did find all steps with $N_{\max } \leqslant 14$ ). This leads to the conjecture that there are no states which admit a maximal set of $d^{2}-1$ orthogonal unitaries in $d$ dimensions. We have proved this to be the case in two dimensions, but, due to the increasing size of the numerical problems, we have only been able to check this conjecture numerically for $d=3,4$. This was done by applying the numerical procedure to states which are nearly maximally entangled. When trying to find $d^{2}-2$ unitaries, the procedure gets as close to a root as desired. However, when trying to find $d^{2}-1$ unitary operators, there seems to be a positive finite minimum. The value of this minimum is small, depending on how close the state is to a maximally entangled state. It might be that states which allow for $d^{2}-1$ orthogonal unitaries are very special and were not discovered by our numerical techniques, or only exist in higher dimensions. However, at the very least, the situation and solution space is dramatically different for $N=d^{2}-1$.

It is also interesting to extract from the numerical results the minimal entanglement necessary for having $N$ orthogonal unitaries. These values are easily found from Fig. 1 by finding the points with minimal entanglement within each region. These points are indicated in Fig. 1. In Fig. 2 we compare this quantity with the lower bound of the amount of entanglement derived from the asymptotic channel capacity [5], which, when measured in units of dits, is given by

$$
C \leqslant 1+S(\psi) \text {. }
$$

Therefore, the entanglement is bounded from below by $S(\psi) \geqslant \log _{d} N-1$ edits. It is evident that only when $N$ is a 
multiple of $d$ is this bound achieved by our deterministic schemes. As expected, this shows that from an asymptotic point of view, motivated by the information capacity of the communication protocol, the deterministic procedure is worse than the probabilistic procedure suggested in [5], which does achieve the bound.

While analyzing the numerical results for the threedimensional case we have noticed that the state $\left|\psi_{3}\right\rangle$ [see Eq. (13)], for which a specific construction was presented in the previous section, seems to be the state with minimal entanglement that admits four orthogonal unitaries. This can be seen in Fig. 1. In addition, for dimensions $d=4, \ldots, 7$, we find that among all states for which the numerical procedure yielded $d+1$ orthogonal unitaries, the state $\left|\psi_{d}\right\rangle$ $=\sqrt{(d-1) / d}|00\rangle+\sqrt{(1 / d)}|11\rangle+0 \sum_{i=2}^{d-1}|i i\rangle$ [see Eq. (15)] is the one with minimal entanglement. In $d=3,4,5$ we have established the optimality of $\left|\psi_{d}\right\rangle$ by applying the numerical procedure to all pure states $|\psi\rangle$ with entanglement $S(|\psi\rangle)$ $\leqslant S\left(\left|\psi_{d}\right\rangle\right)$ over a grid of Schmidt coefficients with resolution 0.01 . Indeed, the numerical procedure found $d+1$ orthogonal unitaries only for $\left|\psi_{d}\right\rangle$. Near the optimal state we employed a higher mapping resolution of the order of $10^{-3}$ to locate the optimum accurately. In dimensions $d=6,7$ we established the local optimality of $\left|\psi_{d}\right\rangle$ with the same accuracy. However, establishing it as a global minimum takes a prohibitive amount of computation. We therefore conjecture that the state with minimal entanglement, admitting at least $d+1$ orthogonal unitaries in $d$ dimensions, is $\left|\psi_{d}\right\rangle$.

In a similar manner, we examined the minimally entangled states for which the numerical procedure found a construction of at least $N$ orthogonal unitaries in dimensions $d=3, \ldots, 7$ for $d+1<N<2 d$. Again, we note that for $d$ $=3,4$ we have established the optimality by mapping the entire relevant domain with a resolution of at least 0.01 , while for $d=5,6,7$ we only verified local optimality. Since for $N=2 d$ a geometric construction for a maximally entangled qubit residing in $d$ dimensions exists, we had expected, and indeed found, that all these states have less than one ebit of entanglement. Remarkably, all of these optimal states have only two nonvanishing Schmidt coefficients. Table I shows the value of $\lambda_{0}$ of these states for different values of $N$ and $d$. Since there are only two nonvanishing Schmidt coefficients, specifying $\lambda_{0}$ completely characterizes the state. Inferring from these results, we conjecture that in any dimension $d$, the state with minimal entanglement that admits at least $d+n(n=2, \ldots, d)$ orthogonal unitaries is $\sqrt{d /(d+n)}|00\rangle+\sqrt{n /(d+n)}|11\rangle$. Note that although this data were generated numerically, these values are simple fractions of $N$ and $d$. This suggests that an explicit analytical construction of the unitary operators is possible for these cases as well.

\section{CONCLUSIONS}

Let us first summarize the results of this work. The main results are either proved analytically, or shown by an explicit construction.

(1) We proved that deterministic dense coding with partial entanglement is impossible in two dimensions $(d=2)$.
TABLE I. Values of $\lambda_{0}$ for states with minimal entanglement, such that there exist $N$ (row index) orthogonal unitary transformations in $d$ (column index) dimensions. Numerical data and conjectured behavior are shown. The estimated accuracy of the values is $10^{-3}$. Values for which global optimality is either known $(N=2 d)$, or numerically verified, appear in boldface, while for other values we have only been able to verify local optimality. Note that all values correspond to less than one ebit of entanglement.

\begin{tabular}{|c|c|c|c|c|c|c|c|c|c|}
\hline & 2 & 3 & 4 & 5 & 6 & 7 & $\ldots$ & $\ldots$ & $d$ \\
\hline 3 & & & & & & & & & \\
\hline 4 & $2 / 4$ & $2 / 3$ & & & & & & & \\
\hline 5 & & $3 / 5$ & $3 / 4$ & & & & & & \\
\hline 6 & & $3 / 6$ & $4 / 6$ & $4 / 5$ & & & & & \\
\hline 7 & & & $4 / 7$ & $5 / 7$ & $5 / 6$ & & & & \\
\hline 8 & & & $4 / 8$ & $5 / 8$ & $6 / 8$ & $6 / 7$ & & & \\
\hline 9 & & & & $5 / 9$ & $6 / 9$ & $7 / 9$ & & & \\
\hline 10 & & & & $5 / 10$ & $6 / 10$ & $7 / 10$ & & & \\
\hline 11 & & & & & $6 / 11$ & $7 / 11$ & & & \\
\hline 12 & & & & & $6 / 12$ & $7 / 12$ & & & \\
\hline 13 & & & & & & $7 / 13$ & & & \\
\hline 14 & & & & & & $7 / 14$ & & & \\
\hline$\vdots$ & & & & & & & $\ddots$ & ${ }^{\circ}$ & \\
\hline$d+1$ & & & & & & & & $\ddots$ & $d-1 / d$ \\
\hline$d+2$ & & & & & & & & & $d / d+2$ \\
\hline$\vdots$ & & & & & & & & & $\vdots$ \\
\hline $2 d-1$ & & & & & & & & & $d / 2 d-1$ \\
\hline $2 d$ & & & & & & & & & $d / 2 d$ \\
\hline
\end{tabular}

(2) We showed that deterministic dense coding with partially entangled states is possible for dimensions $d \geqslant 3$ by constructing exact deterministic dense coding schemes for an alphabet size of $2 d$. A necessary condition for the existence of similar schemes for alphabet size $k d(2<k \leqslant d)$ is that it is possible to distill deterministically a $k$-level maximally entangled state from the initial state $|\psi\rangle$ (or equivalently, that $\left.\lambda_{0} \leqslant 1 / k\right)$ (albeit with classical communication).

(3) We showed an explicit dense coding scheme for an alphabet size of $d+1$ in $d$ dimensions for the partially entangled state $\left|\psi_{d}\right\rangle=\sqrt{(d-1) / d}|00\rangle+\sqrt{1 / d}|11\rangle+0 \sum_{i=2}^{d-1}|i i\rangle$. This proves that deterministic dense coding is possible with less than one ebit of entanglement, which means that this approach is not equivalent to the trivial one wherein deterministic concentration transforms a nonmaximal state into an ebit, to be used in the standard dense coding scheme.

In addition, we have used numerical methods to study the problem in the general case. Relying upon these numerical results we conclude the following.

(4) The optimal alphabet size grows in "steps" and can obtain any integer value in the range $\left[d, d^{2}\right]$ with the possible exception of the alphabet size of $d^{2}-1$.

(5) Our numerical data support the conjecture that the state $\left|\psi_{d}\right\rangle$ is the state with minimal entanglement for which deterministic dense coding is possible.

(6) On the basis of our numerical results we conjecture that the state $\sqrt{d /(d+n)}|00\rangle+\sqrt{n /(d+n)}|11\rangle$ is the state with 
minimal entanglement admitting at least $d+n(n=2, \ldots, d)$ orthogonal unitaries in $d$ dimensions (see Table I). This relatively simple form makes us believe that an explicit construction of the unitary operators for this case can be found.

A connection between superdense coding and teleportation has been noted in the past. In [10], a one-to-one correspondence between dense coding schemes and quantum teleportation schemes (for maximal entanglement) was established, and we have already pointed out the similarity between the phase operators presented in Sec. V and the teleportation protocol with partially entangled states discovered independently in [14]. It would be interesting to understand the correspondence between dense coding and teleportation schemes when partial entanglement is used.

Another related topic is the problem of distinguishing unitary operators. The relation between this problem and superdense coding in the maximal case was mentioned in [17]. The conditions for distinguishing a pair of unitary operations have been specified in [18]. It is interesting that our construc- tions provide nontrivial sets of unitary operators which can be perfectly distinguished by a single application of the unitary and a single measurement of a specific partially entangled state.

Finally, it would be interesting to examine whether the construction of a set of unitaries that satisfy the generalized orthogonality condition (7) sheds light on the recent proposal for probabilistic interpretation of evolutions [19].

\section{ACKNOWLEDGMENTS}

S.M. and B.R. acknowledge the support of ISF Grant No. 62/01-1. J.O. is supported by EU Grant No. PROSECCO (IST-2001-39227), and a grant from the Cambridge-MIT Institute as well as ISF Grant No. 129/00-1. Part of this research was conducted during the Benasque session on Quantum Information and Communication, and we thank the town and the organizers for their hospitality.
[1] C. H. Bennett and S. J. Wiesner, Phys. Rev. Lett. 69, 2881 (1992).

[2] S. L. Braunstein and H. J. Kimble, Phys. Rev. A 61, 042302 (2000).

[3] S. Bose, V. Vedral, and P. L. Knight, Phys. Rev. A 57, 822 (1998).

[4] A. Barenco and A. Ekert, J. Mod. Opt. 42, 1253 (1995).

[5] P. Hausladen, R. Jozsa, B. Schumacher, M. Westmoreland, and W. K. Wootters, Phys. Rev. A 54, 1869 (1996).

[6] S. Bose, M. B. Plenio, and V. Vedral, J. Mod. Opt. 47, 291 (2000).

[7] M. Horodecki, A. Sen De, U. Sen, and K. Horodecki, Phys. Rev. Lett. 90, 047902 (2003).

[8] B. Reznik, e-print quant-ph/0203055.
[9] A. Peres Quantum Theory: Concepts and Methods (Kluwer Academic, Dordrecht, 1993).

[10] R. F. Werner, J. Phys. A 34, 7081 (2001).

[11] H. K. Lo and S. Popescu, Phys. Rev. A 63, 022301 (2001); e-print quant-ph/9707038.

[12] M. A. Nielsen, Phys. Rev. Lett. 83, 436 (1999).

[13] F. Morikoshi and M. Koashi, Phys. Rev. A 64, 022316 (2001).

[14] G. Gour, Phys. Rev. A 70, 042301 (2004).

[15] G. Gour (private communication).

[16] A. Horn, Am. J. Math. 76, 620 (1954).

[17] A. M. Childs, J. Preskill, and J. Renes, J. Mod. Opt. 47, 155 (2000).

[18] A. Acin, Phys. Rev. Lett. 87, 177901 (2001).

[19] J. Oppenheim and B. Reznik, Phys. Rev. A 70, 022312 (2004). 